\title{
THE MASS SPECTRUM OF THE WHITE DWARFS IN CATACLYSMIC BINARIES
}

M. Politano'), H. Ritter ${ }^{2}$ ), and R. F. Webbink ${ }^{1}$,

1) University of Illinois at Urbana-Champaign, U.S.A.

2) Universitäts-Sternwarte München, F.R.G.

\section{ABSTRACT}

Using the theoretically predicted mass spectrum of the white dwarfs (WDs) in zero-age cataclysmic binaries (ZACBs) by Politano and Webbink (1988), we examine to what extent observational selection can account for the observed high masses of the WDs in cataclysmic binaries ( $\mathrm{CBs}$ ).

\section{INTRODUCTION}

It has long been suspected that the high masses of the WDs observed in CBs (for a compilation of WD masses in CBs see, e.g., Ritter 1987a; for a discussion, Ritter 1987b) are due to some selection effect. That observational selection could, in fact, be the answer to the puzzle of the WD masses in CBs has first been shown by Ritter and Burkert (1986), Ritter and Özkan (1986), and Ritter (1986), hereafter referred to respectively as papers I, II and III. In these papers it was shown that flux limitation introduces a very strong bias in favor of observing CBs with highmass WDs. Whereas the strength of the selection effect, expressed in terms of the selection function defined in paper $I$, is independent of the intrinsic distribution of the WD masses, $p_{1,1}$, the corresponding mass spectrum of a flux-limited sample, $\mathrm{p}_{1,0}$, does of course depend on $\mathrm{p}_{1,1}$. Therefore, the question whether the selection effect is strong enough cannot be answered without knowing $p_{1,1}$. The estimates made in papers I and II using the mass spectrum of single white dwarfs, $i . e ., p_{1, i}=p_{S W D}$, showed that the selection effect is in fact strong enough to account for the observed WD masses in CBs if $p_{1, i}$ is not too different from $p_{S W D}$.

In the meantime, work done by Politano and Webbink (1988) provides, for the first time, a detailed and self-consistent theoretical prediction of the intrinsic mass distribution of the WDs in ZACBs, $\mathrm{P}_{Z A C B}$. In this paper we use $\mathrm{p}_{Z A C B}$ in place of $\mathrm{P}_{\mathrm{S} \text { WD }}$ to examine to what extent earlier conclusions about the importance of observational selection remain valid. 


\section{THE INTRINSIC MASS SPECTRUM OF THE WHITE DWARFS}

Politano and Webbink (1988) compute the distribution functions of the orbital parameters, i.e., of the WD mass $M_{1}$, the mass ratio $q-M_{2} / M_{1}$, and the orbital period $P$, for ZACBs that form after a time $t$ of constant star formation. However, the selection problem does not directly involve the distribution functions for newly formed CBs, but rather the intrinsic mass spectrum $p_{1,1}$ of the WDs of the present, secularly evolved, $C B$ population. The distribution functions characterizing the ZACBs and $p_{1, i}$ are related in a rather complicated way. However, under three not unreasonable assumptions, this relation simplifies considerably. The assumptions are: 1) Observational selection through flux-limitation is insensitive to the intrinsic mass spectrum of the secondaries of ZACBs. Computations in paper I showed this to be the case. 2) The time scale for the secular evolution of a typical $C B$ is short compared to the time scale on which $\mathrm{p}_{Z_{A} C_{B}}$ changes. Since the intrinsically brightest systems, which are predominantly seen in a magnitude-limited sample, are also the youngest, i.e., at most a few $10^{8}$ yr past the ZACB stage, this assumption is also reasonably well fulfilled. 3) White dwarf masses in CBs are negligibly affected by secular evolution. With these assumptions, it follows that $p_{1,1}$ is wellapproximated by the current distribution $\mathrm{P}_{\mathrm{ZACB}}$. We take for the latter the distribution $\mathrm{P}_{\mathrm{ZACB}}$ after $10^{10} \mathrm{yr}$ of constant star formation.

TABLE 1: Main characteristics of the intrinsic WD mass spectra $\mathrm{P}_{\mathrm{ZACB}}\left(10^{10} \mathrm{yr}\right)$ and $\mathrm{p}_{\mathrm{SWD}}$

this paper $\quad$ Ritter and Burkert
$(1986)$

\begin{tabular}{llll} 
mass spectrum $p_{1, i}$ & $p_{Z A C B}$ & $p_{S W D}$ \\
Helium WDs & $\begin{array}{l}\text { relative number } \\
\text { mean mass }\end{array}$ & 0.60 & 0.0 \\
\multirow{2}{*}{ CO WDs } & $\begin{array}{l}\text { relative number } \\
\text { mean mass }\end{array}$ & $0.34 \mathrm{M}_{\odot}$ & - \\
& & $0.75 \mathrm{M}_{\odot}$ & 1.0 \\
\hline
\end{tabular}

The main properties of $\mathrm{P}_{Z A C B}\left(10^{10} \mathrm{yr}\right)$ and of $\mathrm{P}_{\mathrm{SwD}}$ are 1isted in Table 1 . The most important difference between $\mathrm{P}_{Z \mathrm{ACB}}$ and $\mathrm{p}_{\mathrm{SWD}}$ is the presence and dominance of helium WDs in $p_{Z A C B}$. However, it is important to note that for $M_{1} \geq 0.6 M_{\odot}, p_{Z A C B}$ and $p_{S W D}$ agree to within a factor of -2 , and that $p_{S W D}$ is flatter than $P_{Z A C B}$ for $M_{1} \geq 0.85 M_{\odot}$.

III. THE METHOD

We compute the mass spectrum $p_{1,0}$ and the expectation value of the WD mass $\left\langle M_{1}\right\rangle$ for a number of flux-limited samples of CBs (defined in Table 2), using $p_{1,1}-p_{Z A C B}\left(10^{10}\right.$ 
yr). Because of the definition of the selection function $s_{1}$ (see paper $I$ ), we have

$$
p_{1,0}=S_{1} p_{1,1}
$$

and

$$
\left\langle\mathrm{M}_{1}>=\int \mathrm{Mp}_{1,0} \mathrm{dM}\right.
$$

Since selection is independent of $p_{1, i}$, we may use for $s_{1}$ the selection functions computed in papers $I$ and II.

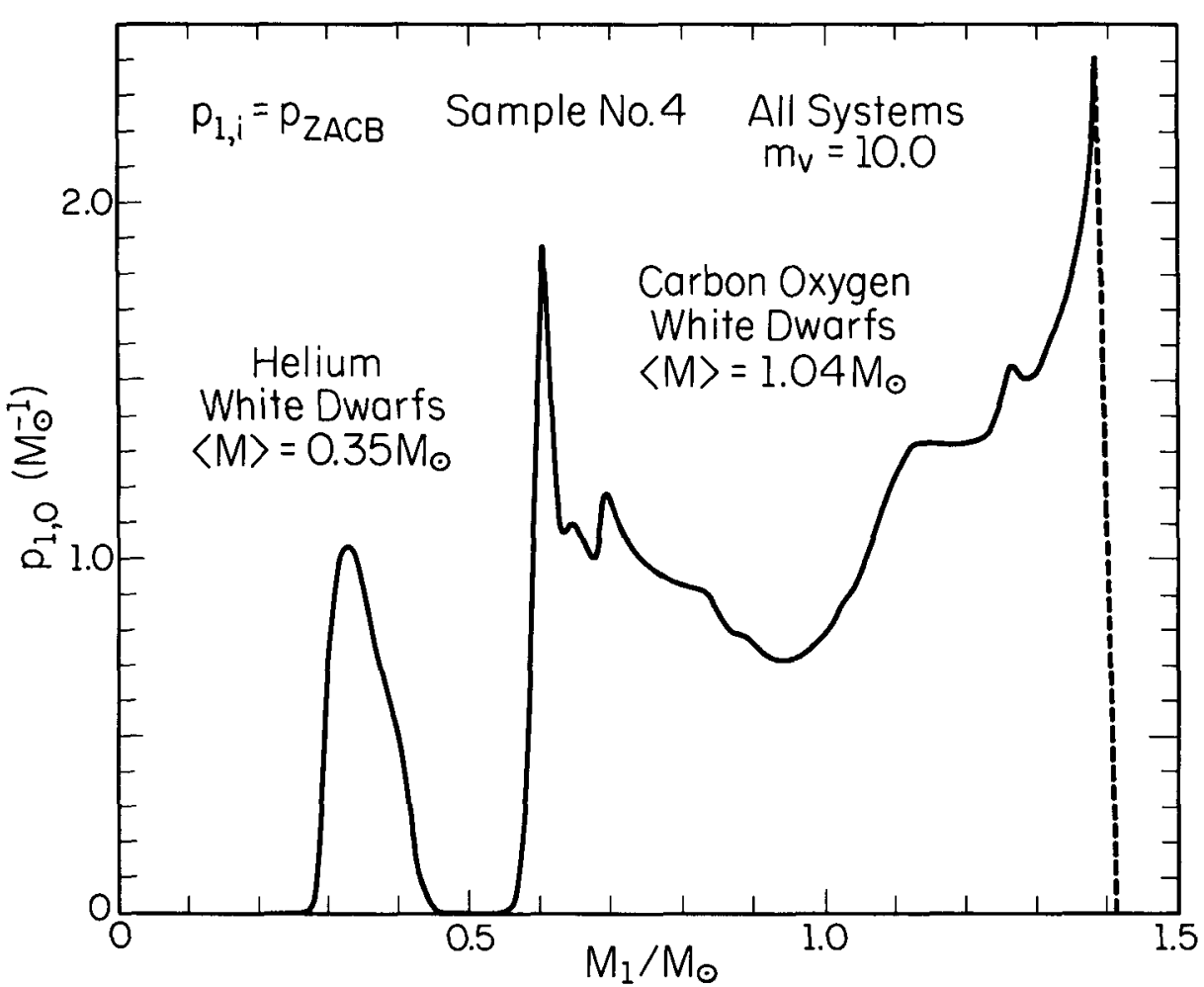

Figure 1: Normalized mass spectrum $p_{1,0}\left(M_{1}\right)$ of the WDs of sample No. 4 defined in Table 2 .

IV. RESULTS

Table 2 lists the defining characteristics and the resulting properties of $p_{1,0}$ of 5 different magnitude-limited samples computed with $p_{1, i}=p_{2 A C B}$. For comparison, we list also the corresponding results obtained in paper $I$ using $p_{1, i}-P_{S} D^{\text {. In order }}$ to illustrate the strength of the selection effect, we show in Fig. 1 the function $p_{1,0}$ for sample No. 4 of Table 2 . The corresponding function $p_{1,1}=p_{Z A C B}$ may be 


\begin{tabular}{|c|c|c|c|c|c|c|c|}
\hline \multicolumn{3}{|c|}{$\begin{array}{l}\text { Defining characteristics of } \\
\text { magnitude-limited samples }\end{array}$} & \multicolumn{4}{|c|}{$p_{1,1}=p_{Z A C B}$} & \multirow{2}{*}{$\begin{array}{c}\mathrm{P}_{1,1}=\mathrm{P}_{\mathrm{SWD}} \\
\text { CO white dwarfs } \\
<\mathrm{M}_{1}>\end{array}$} \\
\hline No. & $\underset{\mathrm{m}_{\mathrm{v}}}{\operatorname{limiting}}$ & $\begin{array}{l}g \text { range of } \\
\text { orbital periods }\end{array}$ & $\begin{array}{l}\text { relative } \\
\text { number }\end{array}$ & $\frac{\left\langle\mathrm{M}_{1}>\right.}{\mathrm{M}_{\odot}}$ & $\begin{array}{l}\text { relative } \\
\text { number }\end{array}$ & $\frac{\left\langle M_{1}>\right.}{M_{\odot}}$ & \\
\hline 4 & 10.0 & al1 systems & 0.09 & 0.35 & 0.91 & 1.04 & 0.98 \\
\hline 12 & 10.0 & below the gap & 0.23 & 0.35 & 0.77 & 0.89 & 0.78 \\
\hline 13 & 10.0 & above the gap & 0.00 & 0.40 & 1.00 & 1.10 & 1.12 \\
\hline 15 & 12.5 & all systems & 0.12 & 0.35 & 0.88 & 0.98 & 0.90 \\
\hline 16 & 15.0 & al1 systems & 0.20 & 0.35 & 0.80 & 0.90 & 0.78 \\
\hline
\end{tabular}

found in Politano and Webbink (1988), and the selection function in paper $I$. The main results may now be summarized as follows (see also Table 2):

- The selection effects introduced through flux-limitation are strong enough to account for the observed high masses of the WDs.

- Using $\mathrm{P}_{1, i}=\mathrm{p}_{\mathrm{ZACB}}$, the results concerning the $\mathrm{CO}$-WDs are qualitatively the same as, and quantitatively similar to, those obtained in papers $I$ and II. Selection is strongest for $M_{1} \geq 0.6 M_{\odot}$, where $p_{Z A C B}$ is very similar to $p_{S W D}$.

- The mean mass of the CO WDs decreases with increasing limiting magnitude $\mathrm{m}_{\mathrm{v}}$.

- No CBs containing a helium WD are expected above the period gap $\left(P \geq 3^{\mathrm{h}}\right)$.

- At $\mathrm{m}_{\mathrm{v}}=10$, about $25 \%$ of the $\mathrm{CBs}$ below the period gap $\left(\mathrm{P} \leq 2^{\mathrm{h}}\right)$ should contain helium WDs.

- The mean mass of the helium WDs in CBs below the gap is $\angle M_{1}>=0.35 M_{\odot}$, and is insensitive to selection effects.

- The fraction of CBs containing helium WDs increases with increasing $m_{v}$.

This research was supported by grants from the NSF (AST 86-16992), DFG, and NATO.

\section{REFERENCES}

Politano, M., Webbink, R.F. 1988, this volume.

Ritter, H. 1986, Astron. Astrophys. 168, 105 (paper III).

Ritter, H., Burkert, A. 1986, Astron. Astrophys. 158, 161 (paper I).

Ritter, H., Özkan, M.T. 1986, Astron. Astrophys, 167, 260 (paper II).

Ritter, H. 1987a, Astron. Astrophys. Supp1. 70, 355.

Ritter, H. 1987b, in: Physical Processes in Comets, Stars and Active Galaxies, W. Hillebrandt, E. Meyer-Hofmeister, and H.-C. Thomas (eds.), Springer Verlag, p. 143. 